\title{
Vertebral compression fractures after stereotactic body radiation therapy: a large, multi-institutional, multinational evaluation
}

\author{
Maha Saada Jawad, MD, ${ }^{1}$ Daniel K. Fahim, MD, ${ }^{2}$ Peter C. Gerszten, MD, ${ }^{3,4}$ John C. Flickinger, MD, ${ }^{3,4}$ \\ Arjun Sahgal, MD, ${ }^{5}$ Inga S. Grills, MD, ${ }^{1}$ Jason Sheehan, MD, ${ }^{6}$ Ronald Kersh, MD, John Shin, MD, ${ }^{8}$ \\ Kevin Oh, MD, ${ }^{9}$ Frederick Mantel, MD, ${ }^{10}$ and Matthias Guckenberger, MD, ${ }^{10,11}$ on behalf of the \\ Elekta Spine Radiosurgery Research Consortium
}

\begin{abstract}
Departments of ${ }^{1}$ Radiation Oncology and ${ }^{2}$ Neurological Surgery, Oakland University William Beaumont School of Medicine, Royal Oak, Michigan; Departments of ${ }^{3}$ Neurological Surgery and ${ }^{4}$ Radiation Oncology, University of Pittsburgh Medical Center, Pittsburgh, Pennsylvania; ${ }^{5}$ Department of Radiation Oncology, Sunnybrook Odette Cancer Centre, Toronto, Canada; ${ }^{6}$ Department of Neurological Surgery, University of Virginia Health System, Charlottesville; ${ }^{7}$ Department of Radiation Oncology, Riverside Medical Center, Newport News, Virginia; Departments of ${ }^{8}$ Neurosurgery and ${ }^{9}$ Radiation Oncology, Massachusetts General Hospital, Boston, Massachusetts; ${ }^{10}$ Department of Radiation Oncology, University Hospital Wuerzburg, Germany; and ${ }^{11}$ Department of Radiation Oncology, University of Zurich, Switzerland
\end{abstract}

OBJECTIVE The purpose of this study was to identify factors contributing to an increased risk for vertebral compression fracture (VCF) following stereotactic body radiation therapy (SBRT) for spinal tumors.

METHODS A total of 594 tumors were treated with spinal SBRT as primary treatment or re-irradiation at 8 different institutions as part of a multi-institutional research consortium. Patients underwent LINAC-based, image-guided SBRT to a median dose of $20 \mathrm{~Gy}$ (range 8-40 Gy) in a median of 1 fraction (range 1-5 fractions). Median patient age was 62 years. Seventy-one percent of tumors were osteolytic, and a preexisting vertebral compression fracture (VCF) was present in $24 \%$ of cases. Toxicity was assessed following treatment. Univariate and multivariate analyses were performed using a logistic regression method to determine parameters predictive for post-SBRT VCF.

RESULTS At a median follow-up of 10.1 months (range $0.03-57$ months), $80 \%$ of patients had local tumor control. At the time of last imaging follow-up, at a median of 8.8 months after SBRT, $3 \%$ had a new VCF, and $2.7 \%$ had a progressive VCF. For development of any (new or progressive) VCF following SBRT, the following factors were predictive for VCF on univariate analysis: short interval from primary diagnosis to SBRT (less than 36.8 days), solitary metastasis, no additional bone metastases, no prior chemotherapy, preexisting VCF, no MRI used for target delineation, tumor volume of $37.3 \mathrm{~cm}^{3}$ or larger, equivalent 2-Gy-dose $\left(\mathrm{EQD}_{2}\right)$ tumor of $41.8 \mathrm{~Gy}$ or more, and $\mathrm{EQD}_{2}$ spinal cord $\mathrm{D}_{\max }$ of $46.1 \mathrm{~Gy}$ or more. Preexisting VCF, solitary metastasis, and prescription dose of $38.4 \mathrm{~Gy}$ or more were predictive on multivariate analysis. The following factors were predictive of a new VCF on univariate analysis: solitary metastasis, no additional bone metastases, and no MRI used for target delineation. Presence of a solitary metastasis and lack of MRI for target delineation remained significant on multivariate analysis.

CONCLUSIONS A VCF following SBRT is more likely to occur following treatment for a solitary spinal metastasis, reflecting a more aggressive treatment approach in patients with adequately controlled systemic disease. Higher prescription dose and a preexisting VCF also put patients at increased risk for post-SBRT VCF. In these patients, pre-SBRT cement augmentation could be considered to decrease the risk of subsequent VCF.

http://thejns.org/doi/abs/10.3171/2015.10.SPINE141261

KEY WORDS radiosurgery; spinal tumors; stereotactic body radiotherapy; spine SBRT; vertebral fractures; oncology

ABBREVIATIONS ASIA = American Spinal Injury Association; $D_{\max }=$ maximum dose; $\mathrm{EQD}_{2}=$ equivalent 2-Gy dose; $\mathrm{MDACC}=\mathrm{MD}$ Anderson Cancer Center; $\mathrm{MSKCC}=$ Memorial Sloan Kettering Cancer Center; SBRT = stereotactic body radiation therapy; SINS = Spinal Instability Neoplastic Score; VCF = vertebral compression fracture. SUBMITTED December 19, 2014. ACCEPTED October 27, 2015.

INCLUDE WHEN CITING Published online February 19, 2016; DOI: 10.3171/2015.10.SPINE141261. 
$\mathrm{M}$ ALIGNANCY involving the spinal column is extremely common, with more than 18,000 new cases of spinal metastases diagnosed in North America each year. ${ }^{7,8,15}$ The role of stereotactic body radiation therapy (SBRT) has become well established in the treatment of primary and metastatic tumors of the spine. The term "stereotactic body radiation therapy" implies high-dose-per-fraction radiation (typically more than 5 Gy) delivered to an image-guided target in 1-5 fractions, using conformal radiation techniques. In contrast to conventional radiotherapy, SBRT uses multiple beams to deliver high doses of radiation while sparing nearby healthy structures, particularly the spinal cord, from potentially toxic doses of radiation. ${ }^{1,2,12,27}$

Several studies have demonstrated the safety and efficacy of SBRT, with local tumor control on the order of $70 \%-90 \%, 2,12,27$ as well as durable pain palliation and preservation of neurological function. ${ }^{8,10,23}$ However, major complications, including radiation-induced myelopathy and vertebral compression fractures (VCFs), remain a significant concern. Radiation therapy using conventional fractionation is known to predispose patients to the development of bony fractures in a dose-dependent manner. ${ }^{18,20,21}$ Similarly, a possible association between spinal SBRT and the subsequent development of VCF has been raised. The literature regarding the risk for VCF following spine SBRT has been limited by small patient numbers and has been somewhat contradictory from study to study.

Part of the controversy in the relationship between spine SBRT and subsequent VCF lies in the understanding of the natural progression of metastatic spinal disease and fracture risk associated with tumor progression outside the setting of radiotherapy. The prevalence of VCF in the setting of metastatic spinal disease appears to be between $28 \%$ and $38 \%$ of patients. ${ }^{6,17,19}$ Taneichi et al. have reported that the most important risk factor for VCF in the thoracic and lumbar spine was the percentage of tumor infiltration within the vertebral body. ${ }^{26}$ They therefore proposed that if radiation could prevent progression within the affected vertebral body, fracture risk may actually be lowered instead of raised. ${ }^{26}$ However, osteoradionecrosis after SBRT, as seen on histopathological examination in a recent case study of 2 patients with VCF after SBRT, suggests that radiation has a significant and mechanistically distinct causative role in VCF as well. ${ }^{2}$ The purpose of this study was to identify factors that increase the risk of VCF following treatment with spinal SBRT.

\section{Methods}

A total of 580 patients with 704 tumors from 8 different institutions underwent irradiation as primary treatment or retreatment for a malignant spinal tumor between 2004 and 2013. Any patients treated with more than 5 fractions were excluded from the analysis and are not reported here. The remaining 594 tumors treated with SBRT in 1-5 fractions were included in this study. All 8 institutions were members of the Elekta Spine SBRT Research Consortium, which included 5 centers in the United States, 2 centers in Canada, and 1 center in Germany. Prior to any data collection, each institution was granted approval by its respective research review board. Data were retrospectively collected by each institution, and the entire cohort was reviewed.

\section{SBRT Treatment Planning}

CT-based treatment planning was performed for all patients. Pretreatment MRI was registered to the planning CT for target delineation in $86 \%$ of patients, while FDG-PET was used for the remainder. Treatment delivery was done using intensity-modulated radiation therapy (n $=575)$ or volumetric-modulated arc therapy $(\mathrm{n}=12)$. All patients had identical treatment delivery technology using LINAC-based SBRT with cone-beam CT image guidance and extracranial immobilization. The majority of immobilization was done with Elekta's BodyFIX (78\%), mask (13\%), mask plus BodyFIX (5\%), or other device (4\%). Online correction for set-up errors was performed using the robotic HexaPod (Elekta) with 6 degrees of freedom.

Tumor and treatment volumes, prescription doses, and dose constraints were defined by each institution according to individual institutional guidelines. Spinal cord dose constraints varied among the institutions. Overall, patients were treated with a median prescription dose of $20 \mathrm{~Gy}$ (range 8-40 Gy) in a median of 1 fraction (range 1-5 fractions). The median maximum-point dose delivered to the spinal cord for the entire cohort was low, with a median of 10 Gy (range 0.1-33.9 Gy). To compare SBRT doses to conventional fractionation, the equivalent 2-Gy dose $\left(\mathrm{EQD}_{2}\right)^{14}$ was calculated for both tumors and spinal cord, assuming an $\alpha / \beta$ ratio of 10 and 2 for tumors and spinal cord, respectively. The median $\mathrm{EQD}_{2}$ for tumors was 36 Gy (range 12-81.1 Gy), and the median EQD 2 spinal cord maximum dose $\left(\mathrm{D}_{\max }\right)$ was $21.6 \mathrm{~Gy}$ (range 0.1-112.7 Gy). Treatment characteristics can be seen in Table 1 .

\section{Toxicity Assessments}

Toxicity was defined according to the Common Terminology Criteria for Adverse Events version 3.0 and was recorded at each follow-up visit. Acute toxicity was defined as side effects within 6 months of treatment completion, and scores for acute dysphagia, dermatitis, and pain were recorded. Chronic toxicity was defined as experiencing side effects more than 6 months following treatment completion. Chronic pain scores were recorded.

Imaging studies (MRI, CT, and/or PET-CT scans) were obtained at baseline and at each follow-up visit following SBRT. Routine follow-up imaging was performed every 3 months. Baseline CT and/or MRI studies were assessed to determine whether a preexisting VCF was present, based

TABLE 1. Treatment characteristics

\begin{tabular}{ll}
\hline \multicolumn{1}{c}{ Characteristic } & \multicolumn{1}{c}{ Median (range) } \\
\hline Total no. fractions & $1(1-5)$ \\
\hline Maximum spinal cord point dose (Gy) & $10(0.1-33.9)$ \\
\hline Prescription dose to target vol (Gy) & $20(8-40)$ \\
\hline Vol of target vol $\left(\mathrm{cm}^{3}\right)$ & $35(0-540.7)$ \\
\hline $\mathrm{EQD}_{2} / 10 \mathrm{SBRT}(\mathrm{Gy})$ & $36(12-81.1)$ \\
\hline $\mathrm{EQD}_{2} / 2 \mathrm{SBRT} \mathrm{D}_{\max }(\mathrm{Gy})$ & $21.6(0.1-112.7)$ \\
\hline
\end{tabular}


upon characteristic imaging findings and evaluation of the vertebral body height. On follow-up imaging, patients were evaluated for tumor progression or control, development of radiation-induced myelopathy, and the presence of a new or worsening VCF. Post-SBRT VCF was defined as either a new or progressive fracture as compared with pre-SBRT imaging. New fractures were defined if imaging demonstrated a VCF that did not exist before SBRT. Progressive fractures were defined as worsening VCF in a treated lesion in which a pre-SBRT fracture was identified. Clinical symptoms were not required for fracture diagnosis. For determination of local control, post-SBRT imaging was carefully reviewed. On CT scanning, progressive lesions were evaluated based on the extent of the soft tissue component as well as the presence of bony destruction, in comparison with the appearance on pre-SBRT images. On PET-CT imaging, a reduction in maximum standardized uptake value on the post-SBRT scan as compared with the pre-SBRT scan was considered response.

\section{Statistical Analysis}

All time intervals were calculated from the date of SBRT completion. Statistical analyses were performed with SPSS version 20 (SPSS, Inc.). All statistical tests were 2 sided. Univariate analysis was performed to determine factors predictive for VCF using a logistic regression. For treatment-related variables (i.e., maximum spinal cord dose, prescription dose to high-dose target volume, volume of high-dose target volume, $\mathrm{EQD}_{2}$ tumor, and $\mathrm{EQD}_{2}$ spinal cord $\left.\mathrm{D}_{\max }\right)$, cutoff points for each value were chosen based on receiver operating characteristic analyses. All variables that were statistically significant or trended to significance in the univariate analysis were included in the multivariate analysis. Multivariate analysis was also performed using a logistic regression with the stepwise forward method. A p value $\leq 0.05$ was considered statistically significant.

\section{Results}

\section{Patient Characteristics and Clinical Outcomes}

Median follow-up for the entire cohort was 10.1 months (range 0.03-57 months). Median imaging follow-up was 8.8 months (range $0-57$ months). A total of 541 patients (91\%) had follow-up imaging available for review. At the time of last imaging follow-up, local tumor control was present in $80 \%$ of these patients $(n=474)$. Local control was verified by MRI in $57 \%$ of patients, CT imaging in $23 \%$ of patients, and PET-CT imaging in 10\%. At the time of analysis, 54\% $(\mathrm{n}=319)$ of patients were dead. Pretreatment patient and tumor characteristics are summarized in Table 2. The median interval of time between diagnosis of the primary disease and SBRT was 1.2 months (range $0-44.4$ months). The median age of the cohort was 62 years (range 16-91 years), and approximately half of the patients were female. The median Karnofsky Performance Scale score was 80 (range 40-100). Overall, the majority of patients had completely intact neurological function (94\% American Spinal Injury Association [ASIA] Grade $\mathrm{E}$ [normal]) prior to SBRT. Half of the patients had tumors located in the thoracic spine (49\%), with $29 \%$ in the lumbar spine, $16 \%$ in the cervical spine, and $7 \%$ in the sacrum.
TABLE 2. Patient and tumor characteristics

\begin{tabular}{|c|c|}
\hline Characteristic & No. of Cases (\%) \\
\hline \multicolumn{2}{|l|}{ Sex } \\
\hline Male & $309(52)$ \\
\hline Female & $285(48)$ \\
\hline \multicolumn{2}{|l|}{ Primary tumor site } \\
\hline Breast & $140(24)$ \\
\hline Lung & $97(16)$ \\
\hline Colorectal & $25(4)$ \\
\hline Kidney & $98(17)$ \\
\hline Melanoma & $24(4)^{\prime}$ \\
\hline Prostate & $49(8)$ \\
\hline Other & $161(27)$ \\
\hline \multicolumn{2}{|l|}{ Neurological status ${ }^{16}$} \\
\hline ASIA Grade E & $561(94)$ \\
\hline ASIA Grade A-D & $33(6)$ \\
\hline \multicolumn{2}{|l|}{ Location of spine tumor } \\
\hline Cervical spine & $95(16)$ \\
\hline Thoracic spine & $283(48)$ \\
\hline Lumbar spine & $173(29)$ \\
\hline Sacrum & $43(7)$ \\
\hline \multicolumn{2}{|l|}{ Epidural spinal cord compression grade ${ }^{4}$} \\
\hline 0 & $168(28)$ \\
\hline 1a & $109(18)$ \\
\hline $1 b$ & $89(15)$ \\
\hline $1 \mathrm{c}$ & $52(9)$ \\
\hline 2 & $34(6)$ \\
\hline 3 & $21(3)$ \\
\hline Unknown & $121(20)$ \\
\hline \multicolumn{2}{|l|}{ Lesion type } \\
\hline Osteolytic & $420(71)$ \\
\hline Sclerotic & $155(26)$ \\
\hline Unknown & $19(3)$ \\
\hline Solitary metastasis present & $105(18)$ \\
\hline Visceral metastases present & $285(48)$ \\
\hline Controlled systemic disease & $147(25)$ \\
\hline Prior palliative chemotherapy received & $429(72)$ \\
\hline Prior bisphosphonates received & $148(25)$ \\
\hline Pre-SBRT compression fracture present & $143(24)$ \\
\hline \multicolumn{2}{|l|}{ Pre-SBRT pain status } \\
\hline None & $80(14)$ \\
\hline Mild/moderate & $429(72)$ \\
\hline Severe & $85(14)$ \\
\hline
\end{tabular}

Primary tumor sites included breast, lung, colorectal, kidney, melanoma, prostate, and other, rarer sites. The majority of lesions were osteolytic (71\%), and $24 \%$ of patients in the cohort had a preexisting VCF, as documented on MRI or CT imaging prior to SBRT delivery. Median survival was compared between patients with a solitary metastasis versus those with multiple metastases. Patients with a solitary metastasis were found to have a longer median survival (40 vs 12 months; $\mathrm{p}<0.001$ ).

\section{Surgical Details}

A total of 205 patients (35\%) received surgical treat- 
ment of the radiation treatment site prior to SBRT delivery, which may have included surgical decompression, percutaneous cement augmentation, or instrumentation/ stabilization surgery. Cases considered for pre-SBRT surgical intervention were evaluated by the neurosurgeons at each institution and an operative plan was determined appropriately. Decompressive surgery included a laminectomy (with tumor debulking, as appropriate). Percutaneous cement augmentation included kyphoplasty or vertebroplasty. Instrumentation/stabilization included a laminectomy along with instrumentation for stabilization. If there was no reason for concern regarding spinal instability, decompression alone was performed. Among the 205 patients who underwent pre-SBRT surgical procedures, $60 \%$ $(n=123)$ underwent decompressive surgery, $15 \%(n=30)$ underwent percutaneous cement augmentation, and 25\% $(\mathrm{n}=52)$ underwent instrumentation/stabilization. Following SBRT, a total of 59 patients $(9.9 \%)$ required surgical intervention for progressive disease or progressive neurological decline; of these patients, $40 \%(n=23)$ had open decompressive surgery, $25 \%(n=15)$ had percutaneous cement augmentation, and 35\% ( $\mathrm{n}=21)$ had instrumentation/ stabilization.

\section{Post-SBRT Toxicity}

Overall, low rates of acute toxicity were noted. Rates of Grade 1 dysphagia, dermatitis, and pain were $7 \%, 2 \%$, and $8 \%$, respectively. Only 2 patients had Grade 2 dysphagia, and none had Grade 3 or higher. Less than $1 \%$ and $0 \%$ of patients had Grade 2 and 3 dermatitis, respectively. Five percent of patients had Grade 2 (moderate) pain and $0.3 \%$ had Grade 3 (severe) pain. No patients developed radiation-induced spinal cord myelopathy. At the time of last clinical follow-up, $85 \%$ of patients had completely intact neurological function (ASIA Grade E), and the remaining $15 \%$ had some neurological deficit (ASIA Grade D or less).

\section{Development of VCF Following SBRT}

A total of 34 patients $(5.7 \%)$ had either a new or progressive VCF following SBRT, with a median time to fracture of 3 months (range 1-36 months); $3 \%$ of patients ( $\mathrm{n}=$ 18) developed a new fracture at the spinal SBRT site, and $2.7 \%(\mathrm{n}=16)$ had progression of an existing pre-SBRT VCF. There was no difference in median survival when comparing patients who developed VCF with those who did not (15.3 vs 17.4 months; $p=0.69$ ).

Univariate analysis was performed to determine factors predictive for any VCF (new or progressive) as well as new VCF following SBRT delivery. Tables 3 and 4 show results of the univariate and multivariate analyses, respectively, for any fracture (new or progressive) following SBRT. Univariate analysis revealed that shorter interval from primary diagnosis to SBRT (less than 36.8 days), solitary metastasis, no additional bone metastases, no prior chemotherapy, presence of VCF prior to SBRT, tumor volume of $37.3 \mathrm{~cm}^{3}$ or larger, $\mathrm{EQD}_{2}$ tumor of $41.8 \mathrm{~Gy}$ or more, and $\mathrm{EQD}_{2}$ spinal cord $\mathrm{D}_{\text {max }}$ of $46.1 \mathrm{~Gy}$ or more were all factors predictive of any VCF. The presence of preexisting VCF, a solitary metastasis, and higher prescription
TABLE 3. Univariate analysis of any (new or progressive) fracture risk

\begin{tabular}{|c|c|}
\hline Variable & p Value* \\
\hline Age, $<62$ yrs vs $\geq 62$ yrs & 0.10 \\
\hline Sex (male) & 0.88 \\
\hline Karnofsky Performance Scale score (<80 vs $\geq 80$ ) & 0.57 \\
\hline Neurological status (ASIA Grade E vs A-D) & 0.41 \\
\hline $\begin{array}{l}\text { Interval from primary diagnosis to SBRT (<36.8 vs } \\
\quad \geq 36.8 \text { days) }\end{array}$ & 0.04 \\
\hline Solitary metastasis (yes) & 0.001 \\
\hline Additional bone metastases (no) & 0.002 \\
\hline Visceral metastases & 0.89 \\
\hline Controlled systemic disease & 0.09 \\
\hline Initial curative treatment of primary disease & 0.31 \\
\hline Prior palliative chemotherapy (no) & 0.04 \\
\hline Prior bisphosphonates & 0.29 \\
\hline Pre-SBRT pain present & 0.48 \\
\hline \multicolumn{2}{|l|}{ No. of vertebral bodies treated } \\
\hline$>1$ vs 1 & 0.88 \\
\hline$>2$ vs 1 or 2 & 0.36 \\
\hline Osteolytic lesion & 0.64 \\
\hline Pre-SBRT compression fracture & 0.003 \\
\hline \multicolumn{2}{|l|}{ Pre-SBRT surgery } \\
\hline Decompression & 0.51 \\
\hline Percutaneous cement augmentation & 0.31 \\
\hline Instrumentation/stabilization & 0.28 \\
\hline \multicolumn{2}{|l|}{ Target delineation } \\
\hline MRI (no) & 0.42 \\
\hline FDG-PET & 0.42 \\
\hline Treatment technique (IMRT vs VMAT) & 0.28 \\
\hline No. of fractions ( $\leq 1$ vs $>1$ ) & 0.29 \\
\hline $\begin{array}{l}\text { Prescription dose to high-dose target vol (<38.4 vs } \\
\geq 38.4 \mathrm{~Gy})\end{array}$ & 0.09 \\
\hline Vol of high-dose target vol $\left(<37.3 \mathrm{vs} \geq 37.3 \mathrm{~cm}^{3}\right)$ & 0.009 \\
\hline $\mathrm{EQD}_{2}$ tumor $(<41.8 \mathrm{vs} \geq 41.8 \mathrm{~Gy})$ & 0.002 \\
\hline $\mathrm{EQD}_{2}$ spinal cord $\mathrm{D}_{\max }(<46.1 \mathrm{vs} \geq 46.1 \mathrm{~Gy})$ & 0.04 \\
\hline Tumor progression & 0.34 \\
\hline
\end{tabular}

IMRT = intensity-modulated radiation therapy; VMAT = volumetric-modulated arc therapy.

* Boldface type indicates statistical significance.

dose to the target volume (38.4 Gy or more) were predictive on multivariate analysis. The results of the univariate and multivariate analyses for new fractures are reported in Tables 5 and 6 . Factors predictive for new fracture on univariate analysis included solitary metastasis, no additional bone metastases, and no MRI used for target delineation. On multivariate analysis, factors that remained predictive were solitary metastasis and lack of MRI use for treatment-planning target delineation.

\section{Discussion}

These data represent, to our knowledge, the largest reported series evaluating the risk factors for VCF follow- 
TABLE 4. Multivariate analysis of any (new or progressive) fracture risk

\begin{tabular}{lcc}
\hline \multicolumn{1}{c}{ Variable } & Odds Ratio $(\mathrm{Cl})$ & $\mathrm{p} \mathrm{Valu{ } ^ { * }}$ \\
\hline Solitary metastasis & $3.46(1.64-7.28)$ & $\mathbf{0 . 0 0 1}$ \\
\hline Pre-SBRT compression fracture & $2.82(1.36-5.85)$ & $\mathbf{0 . 0 0 5}$ \\
\hline Prescription dose to high-dose target vol & $2.28(1.10-4.73)$ & $\mathbf{0 . 0 0 2}$ \\
\hline Primary tumor site & - & 0.17 \\
\hline Additional bone metastases & - & 0.24 \\
\hline Prior palliative chemotherapy & - & 0.57 \\
\hline Interval from primary diagnosis to SBRT & - & 0.20 \\
\hline Vol of high-dose target vol & - & 0.07 \\
\hline $\mathrm{EQD}_{2}$ tumor & - & 0.56 \\
\hline $\mathrm{EQD}_{2}$ spinal cord $\mathrm{D}_{\max }$ & - & 0.40 \\
\hline
\end{tabular}

$-=$ not applicable.

* Boldface type indicates statistical significance.

ing spinal SBRT. At a median follow-up of 10 months, the overall incidence of a new or progressive VCF was approximately $6 \%$. A large proportion of patients (24\%) had preexisting VCF prior to SBRT, with very few developing progression overall. We found that patients with a solitary metastasis at the time of SBRT were at an increased risk for the development of either new VCF or progressive VCF following treatment, likely explained by the use of more aggressive treatment in patients with a more favorable disease profile because these patients were more likely to live longer. As demonstrated by our data, patients with a solitary metastasis had a significantly longer median survival than those with multiple metastases. We also found that higher prescription dose led to an increased risk for fractures overall, although the effect on de novo fracture alone was not significant. As expected, the presence of a pre-SBRT VCF was also predictive for VCF following SBRT, indicating that the presence of a weakened bone confers a higher risk for fracture. In these patients, preSBRT procedures such as kyphoplasty or other percutaneous cement augmentation could be considered. Finally, lack of MRI planning also led to an increased risk for a new VCF. Although the reasons for this are unclear, this effect could be related to the extent of bony disease. In patients for whom very clear osteolytic abnormalities were seen on CT scans, the use of MRI for target delineation may have been less likely. In addition, lack of MRI, leading to increased risk for VCF, could also be related to the volume of vertebral body irradiated. Target delineation without MRI will more likely include the entire vertebral body if the lesion in question cannot be clearly identified on planning CT scans. Therefore, the use of MRI potentially leads to increased target volume accuracy.

Radiation therapy-induced fractures are known to occur in other areas of the axial or appendicular skeleton, particularly following hypofractionated or accelerated treatment regimens, such as accelerated partial breast irradiation for breast cancer and SBRT for lung cancer. ${ }^{18,20}$ However, they may occur after fractionated radiation therapy as well. Overgaard et al. analyzed the influence of fraction size and dose-response relationships on late bone
TABLE 5. Univariate analysis of new fracture risk

\begin{tabular}{|c|c|}
\hline Variable & $\mathrm{p}$ Value \\
\hline Age, $<62$ yrs vs $>62$ yrs & 0.69 \\
\hline Sex (male) & 0.27 \\
\hline Karnofsky Performance Status score (<80 vs $\geq 80$ ) & 0.43 \\
\hline Neurological status (ASIA Grade E vs A-D) ${ }^{26}$ & 0.43 \\
\hline $\begin{array}{l}\text { Interval from primary diagnosis to SBRT ( }<36.4 \text { days vs } \\
\geq 36.4 \text { days) }\end{array}$ & 0.33 \\
\hline Solitary metastasis (yes) & 0.001 \\
\hline Additional bone metastases (no) & 0.006 \\
\hline Visceral metastases & 0.40 \\
\hline Controlled systemic disease (yes) & 0.07 \\
\hline Initial curative treatment of primary disease & 0.10 \\
\hline Prior palliative chemotherapy & 0.67 \\
\hline Prior bisphosphonates & 0.14 \\
\hline Pre-SBRT pain present & 0.27 \\
\hline \multicolumn{2}{|l|}{ No. of vertebral bodies treated } \\
\hline 1 vs $>1$ & 0.98 \\
\hline 1 or 2 vs $>2$ & 0.35 \\
\hline Osteolytic lesion & 0.32 \\
\hline Pre-SBRT compression fracture & 0.15 \\
\hline \multicolumn{2}{|l|}{ Pre-SBRT surgery } \\
\hline Decompression & 0.08 \\
\hline Percutaneous cement augmentation & 0.32 \\
\hline Instrumentation/stabilization & 0.59 \\
\hline \multicolumn{2}{|l|}{ Target delineation } \\
\hline MRI (no) & 0.02 \\
\hline FDG-PET & 0.51 \\
\hline Treatment technique (VMAT vs IMRT) & 0.09 \\
\hline No. of fractions ( $\leq 1$ vs $>1$ ) & 0.23 \\
\hline $\begin{array}{l}\text { Prescription dose to high-dose target vol (<17.9 vs } \geq 17.9 \\
\text { Gy) }\end{array}$ & 0.44 \\
\hline Vol of high-dose target vol $\left(<37.3 \mathrm{vs} \geq 37.3 \mathrm{~cm}^{3}\right)$ & 0.84 \\
\hline $\mathrm{EQD}_{2}$ tumor (<30.9 vs $\left.\geq 30.9 \mathrm{~Gy}\right)$ & 0.37 \\
\hline$E Q D_{2}$ spinal cord $D_{\max }(<7.4$ vs $\geq 7.4 \mathrm{~Gy})$ & 0.34 \\
\hline
\end{tabular}

* Boldface type indicates statistical significance.

damage in 231 patients treated with postmastectomy radiation therapy. ${ }^{18}$ Patients treated with a higher dose per fraction had a significantly increased incidence of spontaneous rib fracture as compared with those receiving the standard dose regimen (48\% vs 6\%). ${ }^{18}$ Pettersson et al. analyzed both the dose response and volume response of

TABLE 6. Multivariate analysis of new fracture risk

\begin{tabular}{lcc}
\hline \multicolumn{1}{c}{ Variable } & Odds Ratio $(\mathrm{Cl})$ & $\mathrm{p} \mathrm{Value}^{*}$ \\
\hline Solitary metastasis & $4.16(1.6-10.9)$ & $\mathbf{0 . 0 0 4}$ \\
\hline MRI for target delineation & $0.33(0.11-0.98)$ & $\mathbf{0 . 0 4}$ \\
\hline Additional bone metastases & - & 0.67 \\
\hline Controlled systemic disease & - & 0.38 \\
\hline
\end{tabular}

* Boldface type indicates statistical significance. 
radiation-induced rib fractures following SBRT for nonsmall cell lung cancer. ${ }^{20}$ In their cohort, 68 patients were treated with 45 Gy in 3 fractions. Dose-volume histograms were evaluated and any ribs receiving more than $21 \mathrm{~Gy}$ were contoured. The authors found that the risk of radiation-induced rib fracture followed a volume response, with higher risks associated with radiation dose to rib volumes greater than $2 \mathrm{~cm}^{3}{ }^{20}$

While it is clear that certain radiation therapy parameters contribute to vertebral fractures following spinal radiation, some risk factors leading to fracture and the timing of fracture remain controversial. Furthermore, it is inconclusive whether radiation confers a greater risk for VCF than the progression of metastatic spine disease itself. Overall, patients receiving conventionally fractionated radiation (ranging from 20 Gy in 5 fractions to $30 \mathrm{~Gy}$ in 10 fractions) for spinal metastases are at a low risk for VCF, with a rate around $3 \%$, as reported in the literature. ${ }^{8}$ With growing interest in and practice of spinal SBRT, concerns about VCF incidence are increased, mainly because of the higher radiation dose per fraction delivered to large portions of the vertebral body. Identification of patients at high risk for VCF following spinal SBRT will allow clinicians to counsel patients appropriately, as well as guide surgeons in the use of pre-SBRT instrumentation or cement augmentation to decrease morbidity from VCF. ${ }^{13}$

The rate of VCF observed in our cohort is lower than rates previously reported in published single-institution series, with some differences noted among the factors that may increase the risk for VCF. Rose et al. reported the Memorial Sloan Kettering Cancer Center (MSKCC) experience evaluating the vertebral body fracture risk following spinal SBRT. ${ }^{22}$ Patients exclusively received highdose single-fraction SBRT (range 18-24 Gy; median 24 Gy) to the entire vertebral body. At a median follow-up of 13 months, the rate of fracture progression was 39\% in 71 treated lesions (62 patients), with a median time to fracture of 25 months. In this analysis, lesions between T-10 and the sacrum, lytic lesions, and increased vertebral body replacement with tumor were independent risk factors for vertebral fracture. No significant association was found with obesity, degree of kyphosis, radiation dose, or the use of bisphosphonates. ${ }^{22}$

Boehling et al. at the MD Anderson Cancer Center (MDACC) subsequently reported the incidence of new or progressive VCF in $20 \%$ of 123 treated vertebrae, with a median time to progression of only 3 months. ${ }^{5}$ SBRT dosing practice varied from single to multiple fractions, with a median dose of 27 Gy (range 18-27 Gy). Patients who had received previous spinal irradiation were also included in the study cohort. The researchers reported that age greater than 55 years, presence of preexisting fracture, higher baseline pain scores, and larger lytic lesions were independent risk factors for fracture. Existing fractures prior to treatment were associated with higher initial pain scores but did not correlate with pain scores following SBRT. Bisphosphonate use was also not associated with fracture risk, whereas obesity was found to have a protective effect. In contrast to the prior MSKCC study, Boehling et al. found that tumor location was not a risk factor for $\mathrm{VCF} .{ }^{5}$

Cunha et al. evaluated the experience of the University of Toronto, reporting a fracture rate of $11 \%$ for 167 spinal segments, with a mean time to fracture of 3.3 months, and a 1-year fracture-free probability of $87 \% .{ }^{9}$ Similar to the MDACC study, the University of Toronto patients were treated with either single- or multifraction SBRT in doses ranging from 8 to $35 \mathrm{~Gy}$, and previously irradiated spinal segments were included. The authors classified each vertebral segment using the Spinal Instability Neoplastic Score (SINS) to determine which patients were stable, potentially unstable, and unstable. As reported by Fisher et al., the SINS criteria consist of location, type of pain versus no pain; spinal misalignment versus normal alignment; presence of baseline VCF versus no collapse; $50 \%$ or more of the vertebral body involved by tumor versus < $50 \%$ involved; type of lesion; and any involvement of the posterolateral elements. ${ }^{11}$ The different components of the SINS, as well as specific clinical and dosimetric factors, were evaluated. The authors observed that patients with lytic tumors, kyphosis/scoliosis, hepatocellular carcinoma and lung histologies, and radiation dose per fraction of more than 20 Gy resulted in a greater likelihood of fracture. ${ }^{9}$

More recently, Sahgal et al. reported results from a multi-institutional study aimed at evaluating the risks and predictive factors associated with VCF following spine SBRT. ${ }^{24}$ Pooling data from the University of Toronto, MDACC, and Cleveland Clinic, this study included 410 spinal segments in 252 patients treated with 1-5 fractions to doses ranging from 8 to $35 \mathrm{~Gy}$. Patients were separated according to the dose per fraction received, which ranged from less than 8 Gy to 24 Gy or more. Each vertebral segment was scored according to the SINS criteria. At a median follow-up of 11.5 months, the authors observed a $14 \%$ overall fracture rate, with $47 \%$ de novo fractures and $53 \%$ progression of an existing fracture. The median and mean times to fracture were 2.5 months and 6.3 months, respectively. Baseline VCF, lytic tumor, spinal deformity, and dose per fraction (highest risk for 24 Gy or more vs 20-23 Gy vs 19 Gy or less) were identified as significant predictors for the development of VCF. ${ }^{24}$

The multi-institutional data by Sahgal et al., ${ }^{24}$ as well as that of the MSKCC, MDACC, and the University of Toronto series, all indicated that the presence of osteolytic spinal disease is an independent risk factor for the development of VCF following spinal SBRT. ${ }^{5,9,22}$ However, our data did not confirm this. An increased risk for VCF in osteolytic tumors, which included progression of preexisting fractures, was not noted in our cohort of patients. There was no association noted between osteolytic disease and the development of a de novo VCF. Osteolytic bone disease causes architectural changes in the trabecular network, including bone loss and decreased mineralization. ${ }^{25}$ This leads to decreased quality of the vertebral bone, leading to an increased risk for VCF. Other studies have been able to assess the size of the lytic lesion or tumor burden present within the vertebral body, or quantify the amount of vertebral body collapse. However, the present series did not. The MDACC series quantified the degree of tumor involvement, using digital volume measurements in addition to evaluating the CT appearance of lesions, to classify them as lytic, sclerotic, or mixed. ${ }^{5}$ Using these CT data, 
they were able to determine the effects of tumor involvement on fracture based upon lytic lesion size, and found that more than $80 \%$ osteolytic tumor within the vertebral body increased the risk for VCF significantly. ${ }^{5}$ Similarly, the MSKCC study quantified the percent of vertebral body involvement using digital volume measurements and estimated the percent vertebral body collapse by comparing the height of the body of interest before and after fracture or by comparing it to vertebral levels cephalad and caudal to the fracture..$^{22}$ In their study, tumor burden was identified as a significant risk factor, with the highest risk noted in tumors with $41 \%-60 \%$ of spinal segment involvement. ${ }^{22}$ In our study, we did not specifically quantify the extent of lytic disease, tumor burden, or amount of vertebral body collapse, which could explain the lack of significance for osteolytic disease in the current analysis. Besides osteolytic disease, multiple other factors, such as age, tumor location, and specific tumor histologies, have also been found to correlate with VCF after SBRT but were not found to be significant in the current study.

Our study population is comparable to that of singleinstitution reports with regard to the inclusion of varying histologies, as well as thoracic spine tumors in approximately half of the cohort. Additionally, our patients were treated with both single-fraction and multifraction SBRT, consistent with previously published data. ${ }^{5,9,22,24}$ As reported by others, ${ }^{5,9,24}$ we found that preexisting fracture confers a greater VCF risk, with no protective effect from bisphosphonates. Factors predictive of VCF that are unique to our study include the effect of a solitary metastasis. This may be related to patients with adequately controlled systemic disease and limited metastases who potentially undergo more aggressive treatment with higher doses and more single-fraction treatments, therefore leading to an increased risk for fracture in a radiation dose-dependent manner. Patients with solitary bone lesions are also more likely to undergo treatment of the entire vertebral body, particularly compared with those with larger, adjacent soft-tissue masses requiring irradiation. As such, our study demonstrated that prescription dose and tumor volume were significantly predictive for VCF overall, although this finding did not hold true when new VCF was analyzed independently. Data from the University of Toronto series demonstrated a radiation dose effect as well; however, a greater risk for vertebral segments treated with 20 Gy or more per fraction was observed in that study, ${ }^{9}$ rather than an association with total radiation dose, as noted in our cohort. In patients with preexisting fractures and, therefore, inherently weaker bone structures, excess radiation dose is felt to play a role in fracture progression. More-conservative doses might be considered in these cases, with an attempt to limit the maximum dose to 38.4 Gy or less in patients at higher risk, based on the current series.

Other considerations for patients with preexisting fractures should include an assessment for surgical intervention prior to SBRT. Recent innovations in spinal surgery have led to the development of less-invasive approaches, including minimally invasive surgery as well as percutaneous procedures. Percutaneous procedures, such as kyphoplasty and vertebroplasty, are available for the relief of pain related to VCF while providing mechanical sta- bility. ${ }^{25}$ In a multicenter, randomized controlled trial, Berenson et al. evaluated the efficacy and safety of balloon kyphoplasty compared with nonsurgical management for painful VCF in 134 patients. $^{3}$ At 1 month, patients who were treated with kyphoplasty had superior functional outcomes, with reduction in back pain and improved quality of life. Kyphoplasty was safe, with a similar rate of adverse events as compared with control subjects. ${ }^{3}$ While existing data show promising results, the possibility of cement extravasation into the neural foramen, ultimately leading to spinal canal compromise, is a potential complication, particularly if patients are not properly selected.

Understanding the role of a combined approach with SBRT is also important, most notably with regard to the timing of each procedure. Gerszten et al. prospectively evaluated the combined use of kyphoplasty and spinal radiosurgery in 26 patients with pathological compression fractures. ${ }^{13}$ Following kyphoplasty using a percutaneous transpedicular technique, patients underwent single-fraction radiosurgery to a mean dose of $18 \mathrm{~Gy}$, with a mean time after kyphoplasty of 12 days. At a median follow-up of 16 months, no radiation-related toxicities or new neurological deficits were observed, and pain improvement was evident in $92 \%$ of the cohort. ${ }^{13}$ The clinical efficacy and safety demonstrated in this study provide a rationale for considering this combination approach in appropriately selected patients, especially with the growing body of data indicating that preexisting fractures increase the risk for further VCF progression.

While the current study does represent a robust clinical data set consisting of a large number of patients, there are limitations to our analysis aside from the inherent shortcomings of a retrospective study. The analysis included variability with regard to treatment dose, fractionation schedules, and normal tissue constraints, since each institution treated patients according to its respective institutional guidelines. Additionally, the definitions of gross tumor volume, clinical target volume, and planning target volume were not standardized, making it unclear whether a link exists between target volume (entire vertebral body vs the involved portion) and increased risk for VCF. Although this heterogeneity in treatment planning was present, all patients were treated similarly, using identical treatment equipment. Similarly, as these data are from multiple institutions with varying practices and available information, several important details could not be included in this analysis that have been shown to be important predictors in prior series examining VCF. Among these are more detailed information regarding the exact tumor size within the bone, the percentage of osteolytic disease, several components of the SINS score, and specific surgical details regarding surgical approaches. Further work is ongoing with respect to surgical approaches and complications, which will be reported at a later time. Also, patients who received previous spinal irradiation or surgical interventions to the SBRT site were included in the analysis, which could confound our findings. However, our data are in concordance with both the University of Toronto and MDACC series, in which prior radiation therapy did not increase the risk for VCF. As re-irradiation is thought to contribute to an increased risk for fracture, fur- 
ther investigation is warranted to determine whether there is a dose- or volume-related effect of pre-SBRT irradiation and to establish if specific cutoff values exist for fracture development.

\section{Conclusions}

To our knowledge, this study represents the largest series to date that specifically evaluated the incidence of VCF after spine radiosurgery. Overall, rates of post-SBRT VCF were relatively low in our study compared with other published series, with minimal rates of other radiationrelated toxicities, supporting the safe use of spinal SBRT in the appropriate setting. VCF following SBRT is significantly more likely to occur following treatment for a solitary spinal metastasis, in patients with preexisting VCF, and with the use of a higher prescription dose. Using MRI for target delineation was found to be protective for development of VCF, and should be done whenever possible for accurate tumor and spinal cord definition. Consideration of pre-SBRT intervention to decrease the risk for new or progressive fractures is warranted in patients with preexisting fracture or who are otherwise at high risk. While higher radiation doses and larger treatment volumes may lead to increased risk for $\mathrm{VCF}$, treating physicians should carefully weigh the risk for VCF versus that of tumor progression due to inadequate radiation dose or volume.

\section{References}

1. Ahmed KA, Stauder MC, Miller RC, Bauer HJ, Rose PS, Olivier KR, et al: Stereotactic body radiation therapy in spinal metastases. Int J Radiat Oncol Biol Phys 82:e803-e809, 2012

2. Al-Omair A, Smith R, Kiehl TR, Lao L, Yu E, Massicotte EM, et al: Radiation-induced vertebral compression fracture following spine stereotactic radiosurgery: clinicopathological correlation. J Neurosurg Spine 18:430-435, 2013

3. Berenson J, Pflugmacher R, Jarzem P, Zonder J, Schechtman K, Tillman JB, et al: Balloon kyphoplasty versus non-surgical fracture management for treatment of painful vertebral body compression fractures in patients with cancer: a multicentre, randomised controlled trial. Lancet Oncol 12:225-235, 2011

4. Bilsky MH, Laufer I, Fourney DR, Groff M, Schmidt MH, Varga PP, et al: Reliability analysis of the epidural spinal cord compression scale. J Neurosurg Spine 13:324-328, 2010

5. Boehling NS, Grosshans DR, Allen PK, McAleer MF, Burton AW, Azeem S, et al: Vertebral compression fracture risk after stereotactic body radiotherapy for spinal metastases. J Neurosurg Spine 16:379-386, 2012

6. Chaichana KL, Woodworth GF, Sciubba DM, McGirt MJ, Witham TJ, Bydon A, et al: Predictors of ambulatory function after decompressive surgery for metastatic epidural spinal cord compression. Neurosurgery 62:683-692, 2008

7. Chao ST, Koyfman SA, Woody N, Angelov L, Soeder SL, Reddy CA, et al: Recursive partitioning analysis index is predictive for overall survival in patients undergoing spine stereotactic body radiation therapy for spinal metastases. Int J Radiat Oncol Biol Phys 82:1738-1743, 2012

8. Chow E, Harris K, Fan G, Tsao M, Sze WM: Palliative radiotherapy trials for bone metastases: a systematic review. J Clin Oncol 25:1423-1436, 2007

9. Cunha MV, Al-Omair A, Atenafu EG, Masucci GL, Letourneau D, Korol R, et al: Vertebral compression fracture (VCF) after spine stereotactic body radiation therapy (SBRT): analysis of predictive factors. Int J Radiat Oncol Biol Phys 84:e343-e349, 2012

10. Degen JW, Gagnon GJ, Voyadzis JM, McRae DA, Lunsden M, Dieterich S, et al: CyberKnife stereotactic radiosurgical treatment of spinal tumors for pain control and quality of life. J Neurosurg Spine 2:540-549, 2005

11. Fisher CG, DiPaola CP, Ryken TC, Bilsky MH, Shaffrey CI, Berven SH, et al: A novel classification system for spinal instability in neoplastic disease: an evidence-based approach and expert consensus from the Spine Oncology Study Group. Spine (Phila Pa 1976) 35:E1221-E1229, 2010

12. Gerszten PC, Burton SA, Ozhasoglu C, Welch WC: Radiosurgery for spinal metastases: clinical experience in 500 cases from a single institution. Spine (Phila Pa 1976) 32:193-199, 2007

13. Gerszten PC, Germanwala A, Burton SA, Welch WC, Ozhasoglu C, Vogel WJ: Combination kyphoplasty and spinal radiosurgery: a new treatment paradigm for pathological fractures. J Neurosurg Spine 3:296-301, 2005

14. Hall EJ, Giaccia AJ: Radiobiology for the Radiologist, ed 7. Philadelphia: Lippincott Williams \& Wilkins, 2012

15. Heron DE, Rajagopalan MS, Stone B, Burton S, Gerszten PC, Dong X, et al: Single-session and multisession CyberKnife radiosurgery for spine metastases-University of Pittsburgh and Georgetown University experience. J Neurosurg Spine 17:11-18, 2012

16. International Spinal Cord Society, American Spinal Injury Association: International Standards for Neurological Classification of Spinal Cord Injury. (http://www. asia-spinalinjury.org/elearning/ASIA_ISCOS_high.pdf) [Accessed December 3, 2015]

17. Maranzano E, Latini P: Effectiveness of radiation therapy without surgery in metastatic spinal cord compression: final results from a prospective trial. Int J Radiat Oncol Biol Phys 32:959-967, 1995

18. Overgaard M: Spontaneous radiation-induced rib fractures in breast cancer patients treated with postmastectomy irradiation. A clinical radiobiological analysis of the influence of fraction size and dose-response relationships on late bone damage. Acta Oncol 27:117-122, 1988

19. Patchell RA, Tibbs PA, Regine WF, Payne R, Saris S, Kryscio RJ, et al: Direct decompressive surgical resection in the treatment of spinal cord compression caused by metastatic cancer: a randomised trial. Lancet 366:643-648, 2005

20. Pettersson N, Nyman J, Johansson KA: Radiation-induced rib fractures after hypofractionated stereotactic body radiation therapy of non-small cell lung cancer: a dose- and volumeresponse analysis. Radiother Oncol 91:360-368, 2009

21. Rodríguez-Ruiz ME, San Miguel I, Gil-Bazo I, Perez-Gracia JL, Arbea L, Moreno-Jimenez M, et al: Pathological vertebral fracture after stereotactic body radiation therapy for lung metastases. Case report and literature review. Radiat Oncol 7:50, 2012

22. Rose PS, Laufer I, Boland PJ, Hanover A, Bilsky MH, Yamada J, et al: Risk of fracture after single fraction image-guided intensity-modulated radiation therapy to spinal metastases. J Clin Oncol 27:5075-5079, 2009

23. Ryu S, Rock J, Rosenblum M, Kim JH: Patterns of failure after single-dose radiosurgery for spinal metastasis. J Neurosurg 101 (Suppl 3):402-405, 2004

24. Sahgal A, Atenafu EG, Chao S, Al-Omair A, Boehling N, Balagamwala $\mathrm{EH}$, et al: Vertebral compression fracture after spine stereotactic body radiotherapy: a multi-institutional analysis with a focus on radiation dose and the spinal instability neoplastic score. J Clin Oncol 31:3426-3431, 2013

25. Sahgal A, Whyne CM, Ma L, Larson DA, Fehlings MG: Vertebral compression fracture after stereotactic body radio- 
therapy for spinal metastases. Lancet Oncol 14:e310-e320, 2013

26. Taneichi H, Kaneda K, Takeda N, Abumi K, Satoh S: Risk factors and probability of vertebral body collapse in metastases of the thoracic and lumbar spine. Spine (Phila Pa 1976) 22:239-245, 1997

27. Yamada Y, Bilsky MH, Lovelock DM, Venkatraman ES, Toner S, Johnson J, et al: High-dose, single-fraction imageguided intensity-modulated radiotherapy for metastatic spinal lesions. Int J Radiat Oncol Biol Phys 71:484-490, 2008

\section{Disclosure}

Dr. Sahgal has received research grants and honoraria for educational seminars from Elekta AB; Dr. Grills owns stock in and is on the board of directors of Greater Michigan Gamma Knife. This research was partially supported through a research grant from Elekta AB, Stockholm, Sweden, with all institutions serving as members of the Elekta Spine SBRT Research Consortium. The data, however, are intellectual property of the individual group members and their sponsoring institutions.

\section{Author Contributions}

Conception and design: Guckenberger, Fahim, Gerszten, Flickinger, Sahgal, Grills, Sheehan, Kersh, Shin, Oh, Mantel. Acquisition of data: Guckenberger, Jawad, Gerszten, Flickinger, Sahgal, Sheehan, Kersh, Shin, Oh, Mantel. Analysis and interpretation of data: Guckenberger, Jawad, Fahim, Gerszten, Grills. Drafting the article: Jawad. Critically revising the article: all authors. Reviewed submitted version of manuscript: all authors. Approved the final version of the manuscript on behalf of all authors: Guckenberger. Statistical analysis: Guckenberger. Study supervision: Guckenberger.

\section{Correspondence}

Matthias Guckenberger, Department of Radiation Oncology, University of Zurich, Raemistrasse 100, Zurich 8091, Switzerland. email: matthias.guckenberger@usz.ch. 